\title{
A utilização de vinhetas na saúde mental: Tradução e adaptação transcultural de uma vinheta de ansiedade social em adolescentes
}

\author{
The use of vignettes in mental health: Translation and cross-cultural \\ adaptation of a social anxiety vignette in adolescents
}

Tânia Morgado (1)

Luís Loureiro (2)

Maria Antónia Rebelo Botelho (3)

(1) Centro Hospitalar e Universitário de Coimbra, Unidade de Investigação em Ciências da Saúde: Enfermagem, Centro de Investigação em Tecnologias e Serviços de Saúde, Portugal

(2) Escola Superior de Enfermagem de Coimbra, Unidade de Investigação em Ciências da Saúde: Enfermagem, Coimbra, Portugal

(3) Escola Superior de Enfermagem de Lisboa, Unidade de Investigação e Desenvolvimento em Enfermagem, Lisboa, Portugal

Recebido: 17/06/2020; Revisto: 10/07/2020; Aceite: 20/10/2020.

https://doi.org/10.31211/rpics.2020.6.2.179

Check for updates

\begin{abstract}
Resumo
Objetivo: Apresentar a tradução e adaptação transcultural de uma vinheta de ansiedade social em adolescentes para o português europeu, para integrar o "Questionário de Avaliação da Literacia em Saúde Mental - QuALiSMental”. Métodos: Realizou-se um estudo metodológico de tradução e adaptação transcultural da vinheta de ansiedade social em adolescentes para o português europeu segundo as etapas: 1) tradução; 2) síntese das traduções; 3) retrotradução; 4) síntese das retrotraduções; 5) painel de peritos, constituído por oito profissionais de diferentes áreas da saúde; 6) cognitive debriefing, integrando seis adolescentes com uma média de idades de 14,33 anos $(D P=0,52)$; 7) revisão e relatório final. Ao longo deste processo, tivemos em conta as considerações éticas. Resultados: Obteve-se uma vinheta de ansiedade social nos adolescentes "João" e "Joana" no português europeu. Salientamos os resultados relativos às etapas: painel de peritos e cognitive debriefing. Foram obtidos os critérios de consenso, entre os peritos, para a equivalência semântica e idiomática, a equivalência experiencial e cultural e a equivalência conceptual. No cognitive debriefing verificou-se $100 \%$ de concordância relativamente à clareza do conteúdo da vinheta no português europeu. Conclusões: Esta vinheta pode ser utilizada na prática clínica, nos diferentes níveis de cuidados, na educação/formação e na investigação. Integrada no QuALiSMental permite a avaliação da literacia em saúde mental sobre a ansiedade em adolescentes em diversos contextos e/ou avaliação da efetividade de intervenções psicoeducacionais nesta área.
\end{abstract}

Palavras-Chave: Saúde Mental; Literacia em Saúde Mental; Ansiedade Social; Adolescentes; Vinhetas; Estudo metodológico.

\section{DI\&D | ISMT}

rpics@ismt.pt https://rpics.ismt.pt
Publicação em Acesso Aberto

(c) 2020. O(s) Autor(es). Este é um artigo de acesso aberto distribuído sob a Licença Creative Commons Attribution, que permite uso, distribuição e reprodução sem restrições em qualquer meio, desde que o trabalho original seja devidamente citado.
Tânia Morgado

CHUC-EPE: Hospital Pediátrico - Serviço de

Pedopsiquiatria

Av. Afonso Romão

3000-602, Coimbra, Portugal

E-mail:218@chuc.min-saude.pt 


\begin{abstract}
Objective: To present the translation and cross-cultural adaptation of a social anxiety vignette in adolescents into European Portuguese and the consequent integration in the Mental Health Literacy Assessment Questionnaire - QuALiSMental. Method: A methodological study of translation and cross-cultural adaptation of a social anxiety vignette in adolescents into European Portuguese was carried out, according to the stages: 1) translation; 2) synthesis of translations; 3) back-translation; 4) synthesis of back-translations; 5) panel of experts with eight professionals from different areas of health; 5) cognitive debriefing integrating six adolescents with an average age of 14.33 years $(S D=0.52) ; 6)$ review and final report. Throughout this process, we have taken into account the ethical considerations. Results: A vignette of social anxiety was obtained in the teenagers "João" and "Joana" in European Portuguese. We highlight the results related to the stages: the panel of experts and cognitive debriefing. Consensus criteria were obtained among experts for semantic and idiomatic equivalence, experiential and cultural equivalence, and conceptual equivalence. In the cognitive debriefing, a 100\% agreement was found regarding the clarity of the vignette's content in the European Portuguese. Conclusions: This vignette can be used in clinical practice at different levels of care, education/training, and research. Integrated with QuALiSMental allows the assessment of mental health literacy on anxiety in adolescents in different contexts and/or assessments of psychoeducational interventions' effectiveness in this area.
\end{abstract}

Keywords: Mental Health; Health Literacy; Social phobia; Adolescent; Vignette; Methodological Study.

\title{
Introdução
}

A palavra "vinheta" corresponde à tradução portuguesa da palavra francesa vignette e a sua utilidade vai desde as artes gráficas e outras áreas da comunicação, às ciências sociais e da saúde, à educação e desenvolvimento profissional e à investigação. Pode ser definida como uma ilustração em palavras, descrevendo um evento, acontecimento, circunstância ou cenário, representativos da realidade ou fictícios, permitindo avaliar crenças, atitudes e/ou comportamentos (Eifler \& Petzold, 2019; Steiner et al., 2016).

A introdução de vinhetas nas ciências sociais e na investigação como estratégia de colheita de dados terá ocorrido nos anos 50 do século XX por Herskovits (1950), numa época de predomínio do paradigma positivista, em que eram elaboradas descrições sistemáticas de situações concretas que se mostravam favoráveis à expressão da opinião dos entrevistados (Torres, 2009). Contemporaneamente, nas ciências da saúde e mais especificamente na área da saúde mental, Woodward (1951) e Star (1952; 1955) foram pioneiros na utilização de vinhetas nas suas investigações sobre o conhecimento e as atitudes da população perante descrições de pessoas com perturbações mentais. Seguiram-se autores como Kluckhohn, Strodtbeck e Ervin-Tripp na década de 60; Rossi, Alexander e Becker na década de 70; Finch nos anos 80; Hughes, Huby, Richman, Mercer, Barter e Renold nos anos 90 e 2000 (Torres, 2009).

No contexto das ciências da saúde adotaram a denominação de vinhetas clínicas e a sua utilização vai desde a educação, a formação e desenvolvimento profissional à intervenção e investigação, sendo utilizadas em diferentes manuais e apresentadas enquanto casos clínicos, inclusive em eventos científicos.

A literatura mostra que a utilização de vinhetas como estratégia de colheita de dados evoluiu ao longo do tempo, podendo ser usadas em diferentes formatos e para diferentes fins (Steiner et al., 2016), em função do tipo (de conteúdo específico e de ancoragem), da forma (face-a-face, por escrito, áudio e/ou vídeo), do contexto e da população a que se destinam.

As vinhetas de conteúdo específico são descrições baseadas em experiências pessoais ou situações hipotéticas, em que introduzem os participantes num cenário que lhes é comum, no qual uma personagem é o foco, e exploram as diferentes dimensões em interação nas respostas dos participantes (Keane et al., 2012). Estas 
vinhetas são particularmente úteis quando o seu conteúdo é manipulado num contexto experimentalmente controlado para analisar os efeitos de variáveis específicas (Keane et al., 2012).

Por sua vez, as vinhetas de ancoragem são breves descrições hipotéticas referentes a situações fictícias relacionadas com um único conceito ou caraterística em estudo, através de itens de autoavaliação e utilizando a mesma escala de resposta, por exemplo uma escala do tipo Likert (Grol-Prokopczyk et al., 2015; King et al., 2004; King \& Wand, 2007). Este tipo de vinhetas é usado para obter comparabilidade entre populações e ajustar diferentes expetativas dos participantes (Keane et al., 2012; King et al., 2004; King \& Wand, 2007) e simultaneamente, pelas suas caraterísticas, aumentam consideravelmente a validade interna e fidelidade dos estudos (Steiner et al., 2016). Investigadores da Organização Mundial de Saúde concluíram que as vinhetas de ancoragem, das estratégias disponíveis, eram as mais promissoras (Grol-Prokopczyk et al., 2015; Keane et al., 2012).

O desenho e validação de vinhetas requer um processo elaborado e sistemático com vista a obtenção de dados adequados em resposta aos objetivos do estudo, exigindo que as situações descritas sejam realistas, concretas, relevantes, estruturadas, compreensíveis, consistentes, concisas, flexíveis e neutras (Steiner et al., 2016; Keane et al., 2012). Assim, as perguntas associadas a vinhetas são mais realistas, menos abstratas e mais atrativas do que as perguntas convencionais (Steiner et al., 2016), podendo ser utilizadas no desenvolvimento de roleplaying para avaliação de opiniões, preferências, intenções ou decisões dos participantes (Rungtusanatham et al., 2011). Devido à sua flexibilidade podem ser usadas como técnica projetiva para evitar respostas socialmente desejáveis ou politicamente corretas ao lidar com tópicos sensíveis (Eifler \& Petzold, 2019; Gourlay et al., 2014; Steiner et al., 2016). Quer na investigação, quer na intervenção, os autores acrescentam outras vantagens da utilização de vinhetas, como facilitar a comunicação entre os profissionais/investigadores e os utentes/participantes, incentivando o relato das suas experiências pessoais através da criação de um ambiente favorável e da identificação com uma terceira pessoa (Eifler \& Petzold, 2019; Findor, 2017; Gourlay et al., 2014; Steiner et al., 2016; Winters \& Weitz-Shapiro, 2013).

Na área da saúde mental e psiquiatria, mais recentemente, vários estudos têm sido desenvolvidos com recurso a vinhetas, nomeadamente na promoção da literacia em saúde mental e redução do estigma, inclusive em crianças, adolescentes e jovens (Attygalle et al., 2017; Clark et al., 2020; Dey et al., 2019; DuPont-Reyes et al., 2020; Hart et al., 2019; Jorm et al., 2007; Loureiro \& Freitas, 2020; Reavley \& Jorm, 2011; Rosa; 2018; Rosa et al., 2014).

Destacamos o trabalho desenvolvido por Anthony Jorm e colaboradores, que definiram o conceito de literacia em saúde mental no final da década de 90 (Jorm et al., 1997), na sequência da definição de literacia em saúde pela Organização Mundial de Saúde (Nutbeam, 1993). Passados alguns anos, Jorm et al. (2007), numa investigação sobre as crenças de primeira ajuda em saúde mental de adolescentes e jovens e respetivos pais, recorreram ao instrumento de colheita de dados Survey of Mental Health Literacy in Young People - Interview Version, utilizando vinhetas sobre a depressão, a depressão com abuso de álcool, a esquizofrenia e a ansiedade social nos adolescentes "John" e "Jenny".

A vinheta de ansiedade social foi incluída pela elevada prevalência de perturbações de ansiedade (Reavley \& Jorm, 2011) e retratava uma situação de ansiedade comum, mas incapacitante, que muitas vezes não chegava a ser diagnosticada (Jorm et al., 2007). Este instrumento de colheita de dados foi trazido para Portugal por Loureiro e colaboradores, que procederam à sua tradução, adaptação transcultural e validação para o português europeu (Loureiro, 2015; Loureiro et al., 2012), com as vinhetas de depressão, esquizofrenia e uso 
de álcool, originando a versão portuguesa Questionário de Avaliação da Literacia em Saúde Mental (QuALiSMental).

Mundialmente, aproximadamente $16 \%$ das crianças e adolescentes sofrem de alguma perturbação mental e a prevalência mundial de qualquer perturbação da ansiedade em crianças e adolescentes é de 6,5\% (Organização Mundial de Saúde, 2018). Em Portugal, as perturbações de ansiedade são as perturbações psiquiátricas mais comuns com uma prevalência anual de 16,5\% (Caldas de Almeida et al., 2015), tendo-se verificado o predomínio da ansiedade em 66,3\% dos 1403 adolescentes com idades entre os 13 e os 17 anos que recorreram à urgência de um hospital pediátrico, de 2011 a 2014 (Trinco \& Santos, 2015). Estes dados reforçam a importância da promoção da saúde mental e da literacia, da prevenção das perturbações mentais, incluindo as de ansiedade e da respetiva identificação, avaliação e intervenção, o mais precocemente possível.

Neste contexto, o presente artigo pretende apresentar o processo de tradução e adaptação transcultural da vinheta de ansiedade social em adolescentes, do instrumento Survey of Mental Health Literacy in Young People - Interview Version (Jorm et al., 2007) para o português europeu e consequente integração no QuALiSMental.

\section{Método}

Realizou-se um estudo metodológico de tradução e adaptação transcultural, segundo Beaton et al. (2007), Wild et al. (2005) e Sousa e Rojjanasrirat (2011), da vinheta de ansiedade social nos adolescentes "John" e "Jenny" (Jorm et al., 2007) para o português europeu, que decorreu em junho e julho de 2015.

\section{Etapa 1 - Tradução}

Após a permissão dos autores da vinheta, iniciou-se o processo de tradução da vinheta do inglês para o português europeu por dois tradutores independentes, originando duas traduções: T1 (por tradutor oficial, português, bilingue) e T2 (por tradutor português, bilingue, enfermeira, conhecedora dos objetivos do estudo).

\section{Etapa 2 - Síntese das traduções}

A partir da vinheta original e da comparação com as duas traduções T1 e T2, a investigadora juntamente com os tradutores realizaram uma síntese das traduções produzindo uma tradução comum (T-12), que originou a primeira versão da vinheta no português europeu.

\section{Etapa 3 - Retrotradução}

Partindo da primeira versão da vinheta no português europeu, novamente dois tradutores independentes traduziram a vinheta do português europeu para o inglês: R1 (por tradutor oficial, nativo inglês, bilingue) e R2 (por tradutor nativo inglês, bilingue, enfermeira, conhecedora dos objetivos do estudo).

\section{Etapa 4 - Síntese das retrotraduções}

As duas retrotraduções R1 e R2 foram comparadas com a versão original da vinheta e foram enviadas ao autor para verificação e validação semântica, o qual se manifestou positivamente, recomendando apenas duas alterações. Deste processo resultou a retrotradução R-12, que deu origem à segunda versão da vinheta no português europeu. 


\section{Etapa 5 - Painel de peritos}

Seguidamente foi constituído um painel interdisciplinar de oito peritos, indo ao encontro das recomendações de Beaton et al. (2007) e Sousa e Rojjanasrirat (2011). Os peritos foram selecionados intencionalmente, segundo os seguintes critérios: 1) ser profissional de saúde perito no domínio da saúde mental e/ou ansiedade e/ou adolescência; 2) ter experiência profissional de, pelo menos, cinco anos; 3) possuir mestrado ou doutoramento; 4) ter experiência curricular relevante na área de perícia (desenvolvimento e implementação de projetos, publicações e comunicações orais e/ou pósteres em eventos científicos). Os peritos foram convidados por email, apresentando o objetivo, a metodologia e a clarificação dos conceitos. Este painel de peritos teve como objetivo avaliar a equivalência semântica/idiomática; experiencial/cultural e conceptual entre a versão original e a segunda versão da vinheta no português europeu (Beaton et al., 2007), atribuindo uma pontuação numa escala de Likert de cinco níveis e sendo dada a possibilidade de apresentar sugestões.

Foram definidos os critérios de consenso (adaptado de Fink et al., 1991): a) média aritmética das pontuações igual ou superior a quatro, com pontuação mínima de três em cada item; b) Pelo menos, $87,5 \%$ de consenso dos peritos (7 em 8 peritos) com pontuação $\geq 4$ em cada item. Os resultados obtidos foram devolvidos a todos os peritos. Desta etapa resultou a terceira versão da vinheta no português europeu.

\section{Etapa 6 - Cognitive debriefing}

Tal como recomendado por Wild et al. (2005) e Sousa e Rojjanasrirat (2011), para garantir que a terceira versão da vinheta no português europeu era clara e compreensível para a população em que viria a ser utilizada, realizouse um cognitive debriefing com seis adolescentes a frequentar o nono ano de escolaridade, selecionados intencionalmente. Este número vai ao encontro da sugestão de Wild et al. (2005), de cinco a oito participantes. Dos adolescentes que participaram, 50\% eram do sexo feminino, com uma média de idades de 14,33 anos ( $D P=$ 0,52 ), idêntica à das personagens da vinheta. Foi solicitado aos adolescentes que lessem a terceira versão da vinheta no português europeu, analisassem a sua clareza e, caso sentissem necessidade, apresentassem sugestões de melhoria. Como critério de consenso, teria de verificar-se pelo menos $80 \%$ de concordância, entre os adolescentes, relativamente ao texto da vinheta e às respetivas alterações sugeridas, tal como referido por Sousa e Rojjanasrirat (2011). Adicionalmente, os adolescentes foram questionados sobre o reconhecimento da situação descrita na vinheta. Desta etapa resultou a quarta versão da vinheta no português europeu.

\section{Etapa 7 - Revisão e relatório final}

Após a revisão final obteve-se a última versão da vinheta no português europeu. Ao longo de todo o processo, tivemos em conta as seguintes considerações éticas: obtenção da autorização do autor da vinheta original para a tradução e adaptação transcultural para o português europeu; a garantia de anonimato dos tradutores envolvidos; a obtenção do consentimento informado dos peritos e garantia de anonimato e confidencialidade; a obtenção do consentimento informado dos adolescentes e respetivos representantes legais para a participação no cognitive debriefing. Por fim, foi realizado o relatório final do processo de tradução e adaptação transcultural da vinheta de ansiedade social em adolescentes para o português europeu, nas versões do "João" e da "Joana". 


\section{Resultados}

A partir da versão original (Quadro 1) da vinheta de ansiedade social nos adolescentes "John" e "Jenny" (Jorm et al., 2007), iremos apresentar a sua versão final no português europeu para os adolescentes "João" e "Joana", dando ênfase aos resultados do painel de peritos e do cognitive debriefing, respetivamente.

\section{Quadro 1}

\section{Versão Original da Vinheta de Ansiedade Social em Adolescentes}

John/Jenny is a 15-year-old living at home with his/her parents. Since starting his/her new school last year he/she has become even more shy than usual and has made only one friend. He/she would really like to make more friends but is scared that he/she'll do or say something embarrassing when he/she's around others. Although John/Jenny's work is OK he/she rarely says a word in class and becomes incredibly nervous, trembles, blushes and seems like he/she might vomit if he/she has to answer a question or speak in front of the class. At home, John/Jenny is quite talkative with his/her family, but becomes quiet if anyone he/she doesn't know well comes over. He/she never answers the phone and he/she refuses to attend social gatherings. He/she knows his/her fears are unreasonable but he/she can't seem to control them and this really upsets him/her.

O painel de peritos foi constituído de acordo com os critérios de seleção e a sua caracterização apresenta-se na Tabela 1.

\section{Tabela 1}

Caracterização dos Peritos ( $N=8)$

\begin{tabular}{|c|c|c|}
\hline \multicolumn{2}{|l|}{ Características } & $M(D P)$ \\
\hline \multicolumn{2}{|l|}{ Idade (anos) } & $49,25(7,52)$ \\
\hline \multicolumn{2}{|c|}{ Tempo de exercício profissional (anos) } & $25,25(6,78)$ \\
\hline & & $n(\%)$ \\
\hline \multirow{3}{*}{ Sexo } & Feminino & $5(62,5)$ \\
\hline & Masculino & $3(37,5)$ \\
\hline & Enfermeiros EESMP (Clínica e/ou Docência) & $4(50,0)$ \\
\hline \multirow[t]{2}{*}{ Atividade Profissional } & Psicólogos (Clínica e/ou Docência) & $3(37,5)$ \\
\hline & Pedopsiquiatra (Clínica) & $1(12,5)$ \\
\hline \multirow{2}{*}{ Formação Académica } & Mestrado & $4(50,0)$ \\
\hline & Doutoramento & $4(50,0)$ \\
\hline
\end{tabular}

Nota. EESMP = Especialistas em Enfermagem de Saúde Mental e Psiquiátrica. 
Foram obtidos os critérios de consenso no painel de peritos (Tabela 2). A maioria das alterações sugeridas pelos peritos foram integradas, resultando deste processo, a terceira versão da vinheta no português europeu.

\section{Tabela 2}

\section{Níveis de Consenso no Painel de Peritos}

\begin{tabular}{lcc}
\hline Equivalência & Média (DP) & \% (Pontuação $\geq 4)$ \\
\hline Equivalência Semântica e Idiomática & $4,75(0,49)$ & 100 \\
Equivalência Experiencial e Cultural & $4,63(0,53)$ & 100 \\
Equivalência Conceptual & $4,69(0,63)$ & 87,5 \\
\hline
\end{tabular}

No cognitive debriefing, após a leitura e análise da terceira versão da vinheta no português europeu pelos adolescentes, verificou-se $100 \%$ de concordância relativamente à clareza do seu conteúdo. Referiram que compreenderam o significado e o sentido de todas as palavras e não apresentaram sugestões. Relativamente ao esclarecimento de algumas questões colocadas pelos peritos: 1) manifestaram preferência pela palavra "exagerados" em vez de "irracionais"; 2) concordaram com a referência ao telefone, apesar de reconhecerem que atualmente os adolescentes utilizam mais frequentemente o telemóvel. Relativamente ao reconhecimento da situação descrita na vinheta, $50 \%$ dos adolescentes consideraram que o(a) adolescente apresentava "falta de confiança"; 33,33\% referiram "ansiedade" e 16,6\% referiram "medo de comunicar"; "stress"; "timidez"; "dificuldade nas relações interpessoais" e "problemas da adolescência". Deste processo resultou a quarta versão da vinheta no português europeu, que após revisões finais deu origem à versão final (Quadro 2).

\section{Quadro 2}

\section{Versão final da Vinheta de Ansiedade Social em Adolescentes no Português Europeu}

$\mathrm{O}(\mathrm{A})$ João/Joana é um(a) adolescente de 15 anos que vive com os pais. Desde que mudou para uma nova escola no ano passado, tornou-se ainda mais tímido(a) do que habitualmente e fez apenas um(a) amigo(a). Ele(a) gostaria de fazer mais amigos, mas tem medo de fazer ou dizer algo embaraçoso quando está com outras pessoas. Embora o desempenho escolar do(a) João/Joana seja razoável, raramente participa nas aulas e fica muito nervoso(a), corado(a), a tremer e sente que pode vomitar se tiver de responder a alguma pergunta ou falar na frente da turma. Em casa, o(a) João/Joana é muito conversador(a) com a família, mas fica calado(a) quando recebem alguém que não conhece bem. Nunca atende o telefone e recusa-se a participar em eventos sociais. Ele(a) sabe que os seus medos são exagerados, mas não consegue controlá-los e isso realmente perturba-o(a). 


\section{Discussão}

O principal resultado deste estudo foi a obtenção e a disponibilização de uma vinheta de ansiedade social em adolescentes no português europeu.

Em relação à metodologia, não se tratando de uma escala ou questionário, optámos por terminar o processo no cognitive debriefing, revisão e relatório final, tal como é sugerido por Wild et al. (2005), apesar de alguns autores alertarem para a necessidade de estudos piloto e estudos de avaliação das características psicométricas de instrumentos (Beaton et al., 2007; Sousa \& Rojjanasrirat, 2011). Relativamente à constituição do painel de peritos, Wild et al. (2005) sugerem aumentar o número de participantes, diversificando os conhecimentos e as experiências. Por exemplo, poderiam ter sido incluídos peritos na área linguística para facilitar o esclarecimento imediato de dúvidas, relacionadas com a equivalência semântica, idiomática e conceptual (Beaton et al., 2007; Sousa \& Rojjanasrirat, 2011; Wild et al., 2005). Da mesma forma, apesar do cognitive debriefing ter sido constituído de acordo com as orientações de Wild et al. (2005), outros autores sugerem um maior número de participantes para diversificar e enriquecer a partilha de opiniões (Sousa \& Rojjanasrirat, 2011).

Em Portugal, o presente estudo surge na continuidade do processo de tradução, adaptação transcultural e validação do instrumento de avaliação da literacia em saúde mental de Jorm et al. (1997) para o português europeu por Loureiro e colaboradores, originando o QuALiSMental (Loureiro, 2015; Loureiro et al., 2012, 2013a, 2013b). Segundo o autor, este instrumento demonstra ser um instrumento válido, com validade de construto e de conteúdo, e fidedigno, com índices de fidelidade satisfatórios (Loureiro, 2015), opinião reiterada por Guo et al. (2018). Pode ser utilizado, quer como instrumento de avaliação da literacia em saúde mental, quer como instrumento de avaliação do impacto das intervenções de promoção da saúde mental em adolescentes e jovens (Loureiro, 2015). No entanto, alguns autores optaram por traduzir e adaptar transculturalmente outros instrumentos de avaliação da literacia em saúde mental em jovens (Loureiro, 2018) ou criar novas vinhetas e/ou novos instrumentos para avaliação da literacia em saúde mental em adolescentes (Campos et al., 2016; Kutcher et al., 2016; O'Connor et al., 2014), inclusive no âmbito do conhecimento específico de enfermagem (Rosa; 2018; Rosa et al., 2014).

Apesar das múltiplas vantagens da utilização das vinhetas, têm vindo a ser referidas algumas desvantagens, nomeadamente no que se refere à validade interna e externa. $O$ caráter multivalente das vinhetas permite uma investigação simultânea de vários fatores e os efeitos da interação entre eles pode ser estimada e testada, sendo que o uso de desenhos experimentais nos estudos com vinhetas garantem-Ihes uma elevada validade interna (Steiner et al., 2016). Alguns autores questionam a capacidade de representatividade da realidade nas vinhetas e a generalização dos resultados, devido aos possíveis desvios nas respostas dos participantes às vinhetas e em contexto real, por exemplo no que se refere à intenção de comportamento e ao comportamento real (Eifler \& Petzold, 2019; Telser \& Zweifel, 2007). Em relação a tópicos sensíveis, as opiniões divergem e, se por um lado, os autores afirmam que o comportamento real dificilmente é determinado com a utilização de vinhetas (Petzold \& Wolbring 2019), por outro lado, quando esses tópicos sensíveis são abordados em estudos experimentais, o viés de desejabilidade social pode ser minimizado com a utilização de vinhetas e com o discurso na terceira pessoa (Eifler \& Petzold, 2019; Gourlay et al., 2014; Steiner et al., 2016). Em particular, o estudo de Winters e WeitzShapiro (2013) relativo ao tópico "corrupção política", recorreu a um estudo experimental e comparou resultados perante vinhetas na terceira e na segunda pessoa que se revelaram idênticos, concluindo que provavelmente perante tópicos moderadamente sensíveis, não será necessária a utilização de vinhetas na terceira pessoa. 
Vários autores preocupados com a validade externa têm desenvolvido estudos que avaliam as escolhas e as decisões dos participantes com recurso simultâneo a vinhetas e ao contexto real (Eifler \& Petzold, 2019; Findor, 2017; Hainmueller et al., 2015; Steiner et al., 2016).

No contexto da ansiedade em adolescentes e jovens, inúmeros autores evidenciam as vantagens da utilização de vinhetas (Attygalle et al., 2017; Clark et al., 2020; Dey et al., 2019; DuPont-Reyes et al., 2020; Hart et al., 2019; Jorm et al., 2007; Loureiro \& Freitas, 2020; Reavley \& Jorm, 2011; Rosa; 2018; Rosa et al., 2014). Apenas um número reduzido de autores alerta para alguns aspetos relacionados com as caraterísticas desenvolvimentais dessa faixa etária, que tidos em conta, incrementam a qualidade dos estudos. Nomeadamente, as possíveis dificuldades de interpretação dos adolescentes e jovens; o facto da sua personalidade estar em desenvolvimento e a grande influência dos pares e do coletivo para o comportamento desejado, em detrimento da posição pessoal e individual (O'Dell et al., 2012).

\section{Conclusão}

Este artigo teve como objetivo apresentar o processo de tradução e adaptação transcultural de uma vinheta de ansiedade social em adolescentes para o português europeu que, além da integração no QuALiSMental, fica disponível para utilização em variados contextos, nomeadamente na prática clínica, na educação/formação e na investigação.

Esta vinheta pode ser utilizada na prática clínica, no âmbito da intervenção na saúde mental da adolescência, seja nos cuidados diferenciados, seja nos cuidados de saúde primários e em contexto escolar, facilitando a intervenção psicoeducacional para promoção da literacia em saúde mental e prevenção da ansiedade, nomeadamente sobre as estratégias de autorregulação, de autoajuda e procura de ajuda profissional especializada.

Na educação e formação, pode ser utilizada quer no âmbito da formação graduada e pós-graduada e de especialização, quer na formação contínua dos profissionais de saúde mental e psiquiatria, na discussão de casos clínicos e no treino de competências com técnicas de simulação e jogos de papéis ou role-playing.

Este estudo tem implicações também para a investigação, evidenciando a necessidade de se desenvolverem estudos experimentais que recorram à utilização desta vinheta integrada no QuALiSMental, para avaliação da literacia em saúde mental sobre a ansiedade dos adolescentes e/ou para avaliação da efetividade de intervenções psicoeducacionais nesta área.

Agradecimentos | Acknowledgements: Um agradecimento especial ao Professor Anthony Jorm pela cedência da vinheta de ansiedade social em adolescentes e a todos os participantes nas diferentes fases do seu processo de tradução e adaptação transcultural para o português europeu.

Conflito de interesses | Conflict of interest: nenhum | none.

Fontes de financiamento | Funding sources: nenhuma | none.

Contributos: TM: Revisão da literatura e aspetos metodológicos; redação e revisão do manuscrito. LL; MARB: Orientação da investigação, contributos na redação e revisão do manuscrito. 


\section{Referências}

Attygalle, U. R., Perera, H., \& Jayamanne, B. D. W. (2017). Mental health literacy in adolescents: Ability to recognise problems, helpful interventions and outcomes. Child and Adolescent Psychiatry and Mental Health, 11, Artigo 38. https://doi.org/fd8x

Beaton, D., Bombardier, C., Guillemin, F., \& Ferraz, M. B. (2007). Recommendations for the cross-cultural adaptation of the DASH \& QuickDASH - Outcome Measures. Institute for Work \& Health, 2007. https://bit.ly/37i88pP

Caldas de Almeida, J. M., Mateus, P., Xavier, M., \& Tomé, G. (2015). Joint action on mental health and wellbeing: Towards community-based and socially inclusive mental health care, European Commission - Portugal situation analysis. EU. https://bit.ly/31jEn4k

Campos, L., Dias, P., Palha, F., Duarte, A., \& Veiga, E. (2016). Development and psychometric properties of a new questionnaire for assessing mental health literacy in young people. Universitas Psychologica, 15(2), 6172. https://bit.ly/3o2DRRV

Clark, L. H., Hudson, J. L., \& Haider, T. (2020). Anxiety specific mental health stigma and help-seeking in adolescent males. Journal of Child and Family Studies, 29, 1970-1981. https://doi.org/fd8z

Dey, M., Marti, L., \& Jorm, A. F. (2019). The Swiss youth mental health literacy and stigma survey: Study methodology, survey questions/vignettes, and lessons learned. European Journal of Psychiatry, 33(2), 72-82. https://doi.org/10.1016/j.ejpsy.2018.12.001

DuPont-Reyes, M. J., Villatoro, A. P., Phelan, J. C., Painter, K., \& Link, B. G. (2020). Adolescent views of mental illness stigma: An intersectional lens. The American Journal of Orthopsychiatry, 90(2), 201-211. https://doi.org/ggm33b

Eifler, S., \& Petzold, K. (2019). Validity aspects of vignette experiments: Expected "what-if" differences between reports of behavioral intentions and actual behavior. Em Lavrakas, P., Traugott, M., Kennedy, C., Holbrook, A., de Leeuw, E., \& West, B. (Eds.), Experimental methods in survey research: Techniques that combine random sampling with random assignment. Wiley. https://doi.org/fd82

Findor, A. (2017). Detecting and reducing intergroup bias: Using vignettes in experimental research. Univerzita Komenského v Bratislave. https://bit.ly/3dEyYJW

Fink, A., Kosecoff, J., Chassin, M. R., \& Brook, R. H. (1991). Consensus methods: Characteristics and guidelines for use. RAND Corporation. https://www.rand.org/pubs/notes/N3367.html

Gourlay, A., Mshana, G., Birdthistle, I., Bulugu, G., Zaba, B., \& Urassa, M. (2014). Using vignettes in qualitative research to explore barriers and facilitating factors to the uptake of prevention of mother-to-child transmission services in rural Tanzania: A critical analysis. BMC Medical Research Methodology, 14, Artigo 21. https://doi.org/f6sh37

Grol-Prokopczyk, H., Verdes-Tennant, E., McEniry, M., \& Ispány, M. (2015). Promises and pitfalls of anchoring vignettes in health survey research. Demography, 52, 1703-1728. https://doi.org/f7v9wc

Guo, S., Armstrong, R., Waters, E., Thirunavukkarasu, S., Alif, S. M., Browne, G. R., \& Yu, X. (2018). Quality of health literacy instruments used in children and adolescents: A systematic review. BMJ Open, 8(6), Artigo e020080. https://doi.org/gdp4wh

Hainmueller, J., Hangartner, D., \& Yamamoto, T. (2015). Validating vignette and conjoint survey experiments against real-world behavior. Proceedings of the National Academy of Sciences, 112(8), 2395-2400. https://doi.org/f63mkr

Hart, L. M., Bond, K. S., Morgan, A. J., Rossetto, A., Cottrill, F. A., Kelly, C. M., \& Jorm, A. F. (2019). Teen mental health first aid for years 7-9: A description of the program and an initial evaluation. International Journal of Mental Health Systems, 13, Artigo 71. https://doi.org/dfh4

Herskovits, M. J. (1950). The hypothetical situation: A technique of field research. Southwestern Journal of Anthropology, 6(1), 32-40. http://www.jstor.org/stable/3628688

Jorm, A. F., Korten, A. E., Jacomb, P. A., Christensen, H., Rodgers, B., \& Pollitt, P. (1997). "Mental health literacy": A survey of the public's ability to recognise mental disorders and their beliefs about the effectiveness of treatment. The Medical journal of Australia, 166(4), 182-186. https://bit.ly/35bkgGv

Jorm, A. F., Wright, A., \& Morgan, A. J. (2007). Beliefs about appropriate first aid for young people with mental disorders: Findings from an Australian national survey of youth and parents. Early Intervention in Psychiatry, 1(1), 61-70. https://doi.org/d9brsz

Keane, D., Lang, A. R., Craven, M. P., \& Sharples, S. (2012). The use of vignettes for conducting healthcare research. Em Duffy, V. G. (Ed.), Advances in human aspects of healthcare (pp. 451-460). CRC Press.

King, G., Murray, G., Salomon, J., \& Tandon, A. (2004). Enhancing the validity and cross-cultural comparability of measurement in survey research. American Political Science Review, 98(1), 191-207. https://doi.org/ck4v67

King, G., \& Wand, J. (2007). Comparing incomparable survey responses: Evaluating and selecting anchoring vignettes. Political Analysis, 15(1), 46-66. https://doi.org/10.1093/pan/mpl011

Kutcher, S., Wei, Y., \& Coniglio, C. (2016). Mental health literacy: Past, present, and future. Canadian Journal of Psychiatry, 61(3), 154-158. https://doi.org/10.1177/0706743715616609

Loureiro, L. (2015). Questionário de Avaliação da Literacia em Saúde Mental - QuALiSMental: Estudo das propriedades psicométricas. Revista de Enfermagem Referência, 4(4), 79-88. https://doi.org/10.12707/RIV14031 
Loureiro, A. (2018). Literacia e saúde mental positiva: Tradução e validação de um instrumento de avaliação em literacia de saúde mental. [Dissertação de Mestrado, Escola Superior de Enfermagem do Porto]. Repositório Comum. http://hdl.handle.net/10400.26/25783

Loureiro, L., Barroso, T., Mendes, A., Rodrigues, M., Oliveira, R., \& Oliveira, N. (2013a). Literacia em saúde mental de adolescentes e jovens portugueses sobre abuso de álcool. Escola Anna Nery, 17(3), $474-481$. https://doi.org/10.1590/S1414-81452013000300010

Loureiro, L. M., \& Freitas, P. M. (2020). Effectiveness of the mental health first aid program in undergraduate nursing students. Revista de Enfermagem Referência, 5(1), e19078. https://doi.org/10.12707/RIV19078

Loureiro, L. M., Jorm, A. F., Mendes, A. C., Santos, J. C., Ferreira, R. O., \& Pedreiro, A. T. (2013b). Mental health literacy about depression: A survey of Portuguese youth. BMC Psychiatry, 13. Artigo 129. https://doi.org/gb826s

Loureiro, L. Pedreiro, A., \& Correia, S. (2012). Tradução, adaptação e validação de um Questionário de Avaliação da Literacia em Saúde Mental (QuALiSMental) para adolescentes e jovens portugueses a partir de um focus group. Revista de Investigação em Enfermagem, 25, 42-48. https://bit.ly/34770bE

Nutbeam, D., Wise, M., Bauman, A., Harris, E., \& Leeder, S. (1993). Goals and targets for Australia's health in the year 2000 and beyond: Report prepared for the Commonwealth Department of Health, Housing \& Community Services [Relatório]. Australia. Department of Health, Housing and Community Services.

O'Connor, M., Casey, L., \& Clough, B. (2014). Measuring mental health literacy-a review of scale-based measures. Journal of Mental Health, 23(4), 197-204. https://doi.org/fd83

O’Dell, L, Crafter, S., Abreu, G., \& Cline, T. (2012). The problem of interpretation in vignette methodology in research with young people. Qualitative Research, 12(6) 702-714. https://doi.org/fd84

Organização Mundial de Saúde. (2018). Adolescent mental health. Fact sheets. https://bit.ly/3dGy0go

Petzold, K., \& Wolbring, T. (2019). What can we learn from factorial surveys about human behavior? A validation study comparing field and survey experiments on discrimination. Methodology: European Journal of Research Methods for the Behavioral and Social Sciences, 15(1), 19-30. https://doi.org/10.1027/1614-2241/a000161

Reavley, N. J., \& Jorm, A. F. (2011). Recognition of Mental Disorders and Beliefs about Treatment and Outcome: Findings from an Australian National Survey of Mental Health Literacy and Stigma. Australian \& New Zealand Journal of Psychiatry, 45(11), 947-956. https://doi.org/10.3109/00048674.2011.621060

Rosa, A. G. S. (2018). Literacia em saúde mental em adolescentes. Desenvolvimento de um instrumento de avaliação [Tese de Doutoramento, Instituto de Ciências Biomédicas Abel Salazar da Universidade do Porto]. Repositório Aberto da Universidade do Porto. https://hdl.handle.net/10216/113131

Rosa, A., Loureiro, L., \& Sequeira, C. (2014). Literacia em saúde mental de adolescentes: Um estudo exploratório. Revista Portuguesa de Enfermagem de Saúde Mental, (spe1), 125-132. https://bit.ly/34fV2Yv

Rungtusanatham, M., Wallin, C., \& Eckerd, S. (2011). The vignette in a scenario-based role-playing experiment. Journal of Supply Chain Management, 47(3), 9-16. https://doi.org/c47hw2

Sousa, V. D., \& Rojjanasrirat, W. (2011). Translation, adaptation and validation of instruments or scales for use in cross-cultural health care research: A clear and user-friendly guideline. Journal of Evaluation in Clinical Practice, 17(2), 268-274. https://doi.org/b4msr3

Star, S. A. (1952, Novembro, 19). What the public thinks about mental health and mental illness [Apresentação de artigo]. The National Association for Mental Health. https://bit.ly/3jgJtEz

Star, S. A. (1955, Novembro, 5). The public's ideas about mental illness illness [Apresentação de artigo]. National Association for Mental Health. Indianapolis, Indiana. https://bit.ly/31oqWQo

Steiner, P., Atzmüller, C., \& Su, D. (2017). Designing valid and reliable vignette experiments for survey research: A case study on the fair gender income gap. Journal of Methods and Measurement in the Social Sciences, 7(2), 52-94. https://doi.org/10.2458/v7i2.20321

Telser, H., \& Zweifel, P. (2007). Validity of discrete-choice experiments evidence for health risk reduction. Applied Economics, 39, 69-78. https://doi.org/10.1080/00036840500427858

Torres, S. (2009). Vignette methodology and culture-relevance: Lessons learned through a project on successful aging with Iranian immigrants to Sweden. Journal of Cross-Cultural Gerontology, 24(1), 93-114. https://doi.org/c2wg2n

Trinco, E., \& Santos, J. (2015). O adolescente com alteração do comportamento no serviço de urgência. Estudo de um quadriénio. Revista Investigação em Enfermagem, 13(1), 18-25. https://bit.ly/3o4fyTA

Wild, D., Grove, A., Martin, M., Eremenco, S., McElroy, S., Verjee-Lorenz, A., \& Erikson, P. (2005). Principles of good practice for the translation and cultural adaptation process for Patient-Reported Outcomes (PRO) measures: Report of the ISPOR task force for translation and cultural adaptation. Value in Health; The Journal of the International Society for Pharmacoeconomics and Outcomes Research, 8(2), 94-104. https://doi.org/c2dxrt

Winters, M., \& Weitz-Shapiro, R. (2013). Lacking information or condoning corruption: When do voters support corrupt politicians? Comparative Politics, 45(4), 418-436. https://www.jstor.org/stable/43664074

Woodward, J. L. (1951). Changing ideas on mental illness and its treatment. American Sociological Review, 16(4), 443454. https://www.jstor.org/stable/2088273 